\title{
Association of heart failure severity with risk of diabetes: a Danish nationwide cohort study
}

\author{
Malene N. Demant • Gunnar H. Gislason • Lars Køber • \\ Allan Vaag • Christian Torp-Pedersen • \\ Charlotte Andersson
}

Received: 1 February 2014 / Accepted: 16 April 2014 / Published online: 22 May 2014

(C) Springer-Verlag Berlin Heidelberg 2014

\begin{abstract}
Aims/hypothesis Heart failure has been suggested to increase the risk of developing diabetes. We investigated the relation between heart failure severity, defined by loop-diuretic dosage, and the risk of developing diabetes in a nationwide cohort of patients with heart failure.

Methods We followed all Danish patients discharged from hospitalisation for first-time heart failure in 1997-2010, without prior use of hypoglycaemic agents, until a claimed prescription for hypoglycaemic agents, death or 31 December 2010. The association of loop-diuretic dosage (furosemide equivalents) 90 days after discharge (study baseline) with risk of diabetes was estimated by multivariate Cox regression models.
\end{abstract}

M. N. Demant $(\bowtie) \cdot$ G. H. Gislason $\cdot$ C. Andersson

Department of Cardiology-post 635, Copenhagen University

Hospital Gentofte, Niels Andersens Vej 65, 2900 Hellerup, Denmark

e-mail: malenedemant@gmail.com

G. H. Gislason • L. Køber • A. Vaag

Faculty of Health and Medical Sciences, University of Copenhagen, Copenhagen, Denmark

G. H. Gislason

National Institute of Public Health, University of Southern Denmark, Copenhagen, Denmark

L. Køber

Department of Cardiology, Heart Centre, Rigshospitalet,

Copenhagen University Hospital, Copenhagen, Denmark

A. Vaag

Department of Endocrinology, Diabetes and Metabolism,

Rigshospitalet, Copenhagen University Hospital,

Copenhagen, Denmark

C. Torp-Pedersen

Institute of Health, Science and Technology, Aalborg University,

Aalborg, Denmark
Results In total, 99,362 patients were included and divided into five loop-diuretic dose groups: 30,838 (31\%) used no loop diuretics; 24,389 (25\%) used $>0-40 \mathrm{mg} /$ day; 17,355 $(17 \%)$ used $>40-80 \mathrm{mg} /$ day; $11,973(12 \%)$ used $>80$ $159 \mathrm{mg} /$ day; and $14,807(15 \%)$ used $\geq 160 \mathrm{mg} /$ day. A total of 7,958 patients $(8 \%)$ developed diabetes. Loop-diuretic dosages were associated with an increased risk of developing diabetes in a dose-dependent manner. Concomitant use of renin-angiotensin system inhibitors (RASis) attenuated the risk ( $p$ value for interaction $<0.0001$ ). Compared with patients using no loop diuretics (group 1), the adjusted HRs (95\% CI) for developing diabetes for groups $2-5$ respectively were 1.16 (1.07, 1.26), 1.35 (1.24, 1.46), $1.48(1.35,1.62)$ and 1.76 $(1.61,1.92)$ with RASi treatment, and $2.06(1.83,2.32), 2.28$ $(2.01,2.59), 2.88(2.52,3.30)$ and $3.02(2.66,3.43)$ without RASi treatment.

Conclusions/interpretation In a nationwide cohort of patients with heart failure, severity of heart failure was associated with a stepwise increased risk of developing diabetes. Increased awareness of risk of diabetes associated with severe heart failure is warranted.

Keywords Cardiac complications - Epidemiology · Prediction and prevention of type 2 diabetes

\section{Abbreviations \\ ATC Anatomical Therapeutical Chemical system \\ NYHA New York Heart Association \\ RASi Renin-angiotensin system inhibitor}

\section{Introduction}

Nearly 35 years ago, the Framingham Heart Study reported that diabetes was associated with worsened prognosis in patients with heart failure [1]. Several studies have since 
confirmed these findings, including more contemporary studies [2-4]. Thus, little or no temporal improvement has been seen in the prognosis associated with diabetes in heart failure despite, for example, intensified glucose-lowering pharmacotherapy. This has led to the hypothesis that diabetes may in some cases be a marker of heart failure severity rather than a causal risk factor for adverse outcomes as such. In this context, chronic heart failure has been shown to be associated with hyperinsulinaemia and increased insulin resistance in a severity-dependent manner [5-7]. The clinical importance of these findings, i.e. the risk of developing overt diabetes according to the severity of the heart failure, has, however, been insufficiently investigated. One study suggested that heart failure increased the risk of developing diabetes in a severity-dependent manner in patients discharged after myocardial infarction [8]. The purpose of the present nationwide study was to investigate whether a relation between loopdiuretic dosages, as a proxy for heart failure severity, and risk of diabetes exists for patients discharged after first-time heart failure hospitalisation, and the prognostic effect of developing diabetes in chronic heart failure.

\section{Methods}

All residents in Denmark are covered by a tax-financed healthcare system. Every citizen has a unique and permanent civil registration number, which can be used to link information at the individual level from nationwide administrative registries. For the present study, we crosslinked four of these registers. In brief, the Danish National Patient Registry holds information on all hospitalisation and discharge diagnoses (coded according to the ICD-10 system since 1994). The diagnosis of heart failure has been validated with a specificity of $99 \%$ and a sensitivity of $29 \%$ in this registry, i.e. heart failure is under-reported, but the diagnosis is very specific [9]. The Danish Registry of Medicinal Product Statistics provides information about all prescriptions dispensed from Danish pharmacies since 1995. Available data include strength, dispensing date and quantity dispensed. Drugs are classified according to the Anatomical Therapeutical Chemical system (ATC). Because the Danish government partially covers drug expenses, the register is accurate and the likelihood of patients obtaining medications from other sources is small [10]. The National Population Register holds information on age, sex and date of death for all individuals.

Population, identification of pharmacotherapy, and comorbidity This study included all Danish citizens $\geq 30$ years of age admitted with first-time heart failure in the period between 1997 and 2010 and alive 90 days after discharge. Patients who had claimed prescriptions of hypoglycaemic agents (ATC code A10) before hospitalisation for heart failure or during the first 90 days after discharge were excluded. Incident diabetes was defined as date of first claimed hypoglycaemic agent (ATC code A10) [11].

Patients were divided into five groups according to the dosage of prescribed loop diuretics based on arbitrary values: group 1, no loop diuretics; group 2, $>0-40 \mathrm{mg} /$ day; group 3, $>40-80 \mathrm{mg} /$ day; group $4,>80-159 \mathrm{mg} /$ day; and group 5 , $\geq 160 \mathrm{mg} /$ day. Mean loop-diuretic dosages were calculated by dividing the number of dispensed tablets by the dispensing time interval, taking up to three consecutively claimed prescriptions into account (in a retrospective manner), a method that has been described in detail previously $[12,13]$. Loopdiuretic dosages have previously been demonstrated to correlate positively with worsened New York Heart Association (NYHA) functional class, lowered peak oxygen uptake and risk of mortality, but not with glomerular filtration rate in patients with heart failure [5]. Usage of selected concomitant cardiovascular pharmacotherapy was defined as at least one claimed prescription in the period 90 days before and up to 90 days after discharge. The following ATC codes were included: $\beta$ blockers, $\mathrm{C} 07$; statins, $\mathrm{C} 10 \mathrm{~A}$; renin-angiotensin system inhibitors (RASis), C09; thiazides, C03A; spironolactone, C03D; calcium antagonists, C08; digoxin, C01AA05; vitamin $\mathrm{K}$ antagonists, B01AA0; aspirin, B01A06; and clopidogrel, B01AC04.

We identified selected comorbid conditions on the basis of a discharge diagnosis for up to 1 year before the heart failure admission date, as previously [8].

Ethics Registers were available in an anonymous set-up, preventing identification of individuals. In Denmark, such studies do not need ethics approval. The study was approved by the Danish Data Protection Agency (No 2007-41-1667).

Statistical analysis Continuous variables were compared using the $t$ test, and discrete variables using the $\chi^{2}$ test. Cause-specific cumulative incidence curves were created using the competing risk macro written by Bergstralh (2004) (www.mayo.edu/research/documents/gmatch.sas/ DOC-10027248, accessed 5 August 2013). Equality over strata was tested using the logrank test. Cox proportionalhazard regression models including death as a competing risk were used to estimate the risk of developing diabetes. All models were adjusted for age, sex, calendar year of hospitalisation, comorbidity and concomitant cardiovascular pharmacotherapy as specified in Table 1. Cox regression models were also used to investigate the subsequent risk of death associated with diabetes. In these analyses, diabetes and age were included as time-dependent variables. Model assumptions were tested and fulfilled unless otherwise indicated. Because the risk of diabetes has previously been suggested to be modified by use of RASis [8], we tested this assumption by including an interaction term in the overall analysis and 
Table 1 Baseline characteristics of patients in the five different loop-diuretic dosage groups

\begin{tabular}{|c|c|c|c|c|c|}
\hline Characteristic & Group 1 & Group 2 & Group 3 & Group 4 & Group 5 \\
\hline Number & 30,838 & 24,389 & 17,355 & 11,973 & 14,807 \\
\hline$\%$ of study population & 31 & 25 & 17 & 12 & 15 \\
\hline Female & $14,222(46)$ & $11,529(47)$ & $8,269(48)$ & $5,923(49)$ & $7,225(49)$ \\
\hline Age at hospitalisation (years), mean $\pm \mathrm{SD}$ & $72.1 \pm 13.5$ & $75.2 \pm 11.9$ & $75.5 \pm 11.7$ & $76.5 \pm 11.5$ & $76.5 \pm 11.5$ \\
\hline \multicolumn{6}{|l|}{ Concomitant diseases } \\
\hline Ischaemic heart disease & $11,727(38)$ & $9,320(38)$ & $6,386(37)$ & $4,398(37)$ & $5,952(40)$ \\
\hline Acute myocardial infarction & $7,902(26)$ & $6,367(26)$ & $4,431(26)$ & $3,104(26)$ & $3,937(27)$ \\
\hline Atrial flutter/fibrillation & $8,476(27)$ & $7,714(32)$ & $5,964(34)$ & $4,171(35)$ & $5,110(35)$ \\
\hline Cerebrovascular disease & $4,678(15)$ & $3,528(14)$ & $2,357(14)$ & $1,723(14)$ & $2,374(16)$ \\
\hline Chronic obstructive pulmonary disease & $4,718(15)$ & $4,708(19)$ & $3,427(20)$ & $2,463(21)$ & $3,611(24)$ \\
\hline Peripheral occlusive artery disease & $1,273(4)$ & $1,056(4)$ & $708(4)$ & $520(4)$ & $801(5)$ \\
\hline Renal disease & $1,061(3)$ & $824(3)$ & $488(3)$ & $476(4)$ & $1,288(9)$ \\
\hline \multicolumn{6}{|l|}{ Concomitant medications } \\
\hline Spironolactone & $4,753(15)$ & $6,636(27)$ & $5,012(29)$ & $3,684(31)$ & $5,771(39)$ \\
\hline $\mathrm{RASi}$ & $17,051(55)$ & $14,484(59)$ & $10,789(62)$ & $7,246(61)$ & $8,682(59)$ \\
\hline Statins & $9,436(31)$ & $6,302(26)$ & $4,306(25)$ & $2,592(22)$ & $3,077(21)$ \\
\hline$\beta$ Blockers & $15,812(51)$ & $12,131(50)$ & $8,777(51)$ & $5,803(48)$ & $6,605(45)$ \\
\hline Aspirin & $17,366(56)$ & $14,185(58)$ & $10,261(59)$ & $7,159(60)$ & $8,783(59)$ \\
\hline Vitamin $\mathrm{K}$ antagonists & $6,653(22)$ & $6,090(25)$ & $4,789(28)$ & $3,145(26)$ & $3,927(27)$ \\
\hline Clopidogrel & $3,474(11)$ & $2,201(9)$ & $1,384(8)$ & $904(8)$ & $990(7)$ \\
\hline Thiazides & $10,292(33)$ & $4,441(18)$ & $2,930(17)$ & 2,275 (19) & $3,227(22)$ \\
\hline Calcium blockers & $8,151(26)$ & $5,922(24)$ & $4,452(26)$ & $3,144(26)$ & $4,392(30)$ \\
\hline Digoxin & $7,814(25)$ & $8,225(34)$ & $6,569(38)$ & $4,826(40)$ & $6,454(44)$ \\
\hline
\end{tabular}

Unless otherwise indicated, values are number (\%). Loop-diuretic dosage groups are as follows: group 1, no loop diuretics; group 2, >0-40 mg/day; group 3, $>40-80 \mathrm{mg} /$ day; group 4, $>80-159 \mathrm{mg} /$ day; group $5, \geq 160 \mathrm{mg} /$ day

subsequently analysed the risks associated with loop-diuretic dosages in patients with and without concomitant RASis by creation of dummy variables (hence, all patients were analysed in the same model). All analyses were performed with SAS version 9.3 (SAS institute, Cary, NC, USA).

\section{Results}

A total of 170,884 patients were hospitalised for heart failure for the first time between 1997 and 2010, of which 121,056 were alive at study baseline (i.e. 90 days after discharge). In total, 119,129 of these patients were aged 30 years or older. Prevalent diabetes resulted in 19,795 (17\%) patients being excluded from analysis, leaving 99,362. Baseline characteristics for the five loop-diuretic dosage groups are shown in Table 1. Prevalence of female sex and age increased with increasing loop-diuretic dosage group (mean \pm SD age $72 \pm 13$ years in group 1 vs $77 \pm 11$ years in group 5; $p<0.0001)$. The proportion of patients with renal disease was overall small, but the disease was more common in group 5 than group 1 ( $9 \%$ vs $3 \%, p<0.0001)$.
Diabetes development In total, 7,958 patients ( $8 \%$ of study population) developed diabetes. Median time to first claimed prescription of a hypoglycaemic agent was 1,080 days (interquartile range 475-2,037).

The proportion of patients who developed diabetes increased with higher loop-diuretic dosage. Among those in the group receiving the highest dosage, the 10-year cumulative risk of developing diabetes exceeded $25 \%$ (Fig. 1). Crude incidence rates for developing diabetes were $1.6(95 \%$ CI $1.5,1.7), 1.9(95 \%$ CI $1.5,2.0), 2.3$ $(95 \%$ CI $2.2,2.4), 2.6(95 \%$ CI $2.5,2.8)$ and $3.0(95 \% \mathrm{CI}$ $2.9,3.2)$ per 100 person-years in groups $1-5$.

HR estimates obtained from the adjusted Cox regression models are presented in Fig. 2. The use of RASis was found to attenuate the risk of diabetes associated with loop-diuretic dosage $(p<0.0001)$.

Explorative analyses HRs associated with loop-diuretic dosage were found to be slightly lower for patients with ischaemic heart disease than for those without: 1.22 (95\% CI 1.10, 1.34) vs 1.29 (95\% CI 1.19, 1.41), 1.38 (95\% CI 1.24, 1.54) vs 1.50 (95\% CI 1.38, 1.64), 1.55 (95\% CI $1.38,1.75)$ vs 1.81 (95\% CI $1.64,1.99)$, and $1.86(95 \%$ CI $1.68,2.07)$ vs 2.00 (95\% CI 


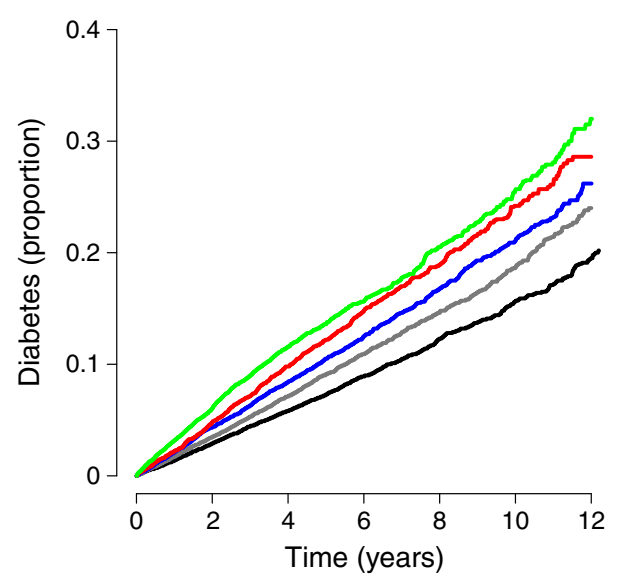

Fig. 1 Cumulative risk of diabetes development according to loopdiuretic dosage. Green line, group 5; red line, group 4; blue line, group 3 ; grey line, group 2; black line, group 1

$1.83,2.20)$ for groups $2-5$ compared with group 1 in patients with and without ischaemic heart disease ( $p$ for interactions $<0.0001$ ). Similarly to the use of RASis, we found lower risk in those who used $\beta$ blockers compared with those who did not: HR 0.86 (95\% CI 0.79, 0.94), 0.97 (95\% CI 0.88, 1.07), $0.91(95 \%$ CI $0.81,1.02)$ and $1.03(95 \%$ CI $0.93,1.14)$ vs 1.60 (95\% CI 1.46, 1.75), 1.66 (95\% CI 1.51, 1.83), 2.03 (95\% CI $1.82,2.26)$ and $2.17(95 \%$ CI $1.96,2.40)$ for groups $2-5$ compared with group 1 ( $p$ for interactions $<0.0001$ ).

Mortality In total, 62,565 (63\%) patients died during the study period.

Adjusted HR for death among those who developed diabetes was $1.16(95 \%$ CI 1.12, 1.19) compared with those who did not. Increasing loop-diuretic dosage was furthermore associated with increasing risk of death: HR 1.14 (95\% CI 1.12, 1.17), 1.17 (95\% CI 1.14, 1.20), 1.29 (95\% CI 1.26, 1.33) and 1.45 (95\% CI $1.41,1.48)$ for groups $2-5$ compared with group 1. The risk associated with diabetes was not dependent on

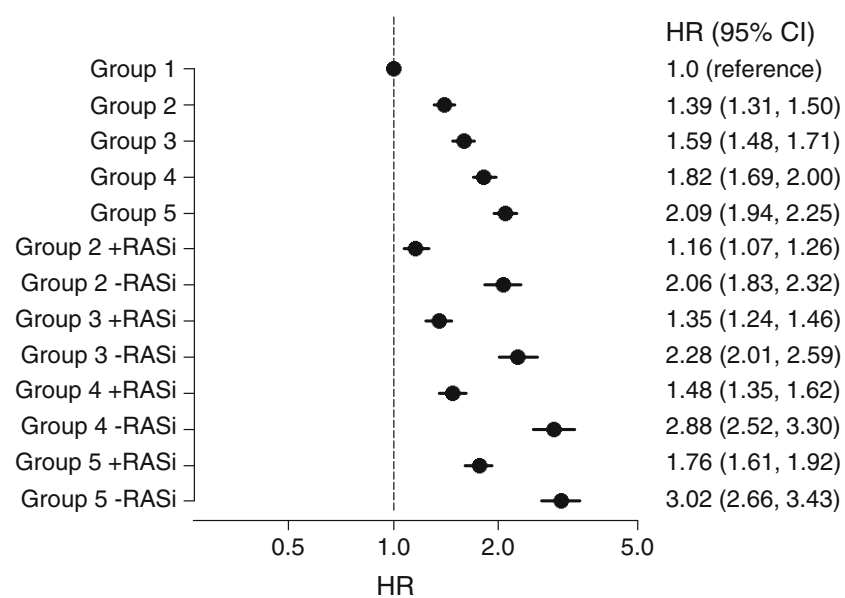

Fig. 2 Risk of diabetes according to loop-diuretic dosage group. HR and $95 \%$ CI overall, with and without RASi treatment. All comparisons were made using group 1 as reference loop-diuretic dosage or RASi use ( $p$ for interactions $=0.21$ and 0.10 ).

\section{Discussion}

This nationwide cohort of patients hospitalised for heart failure showed that increasing loop-diuretic dosage, as estimated at day 90 after discharge and used as a proxy for heart failure severity, was associated with increased risk of developing diabetes in a severity-dependent manner. Furthermore, patients developing diabetes were shown to be at increased risk of dying compared with patients who did not develop diabetes.

In a previous study, we showed that use of loop diuretics as a proxy of heart failure was associated with increased risk of developing diabetes as determined by new prescriptions of glucose-lowering agents among patients discharged after firsttime myocardial infarction [8]. In the present study, we included the entire Danish population discharged after firsttime heart failure and showed that the increased risk of developing diabetes among patients with heart failure was not restricted to patients with a previous myocardial infarction.

This study included patients admitted with heart failure who had not previously been treated with hypoglycaemic agents or loop diuretics. Our data therefore support the notion that heart failure severity makes the patient predisposed to diabetes. Although we cannot exclude the possibility that some of the patients had a pre-existing milder degree of diabetes not requiring glucose-lowering medications, we believe that our approach of defining diabetes as a documented need for glucose-lowering medication as well as our 90-day 'wash-out period' after discharge provides a clear separation of the population with respect to degree of glucose tolerance. Indeed, type 2 diabetes is an arbitrarily defined disease based on consensus criteria and epidemiological associations of prevalence of micro- and macro-vascular complications, and not on distinct aetiological and pathophysiological knowledge with a clear distinction of the state of normal glucose tolerance. Because of the 90-day wash-out period, however, the incidence rates and cumulative incidences were underestimated compared with a regular clinical setting.

From an epidemiological perspective, the poor prognosis associated with diabetes in patients with heart failure has confused researchers for 35 years [1]. Even after adjustment for risk factors such as coronary artery disease and dyslipidaemia, several studies have over the years reported diabetes to be consistently associated with increased mortality. Our data add important insights to the understanding of the mechanisms underlying this poor prognosis, because it might be that the sickest patients are those who develop diabetes. Thus, diabetes may, in part, be a marker of heart failure severity in addition to being a causal risk factor for mortality 
in heart failure cohorts. Although cross-sectional studies have previously shown a higher prevalence of insulin resistance and diabetes with greater heart failure severity, longitudinal analyses have been sparse [6, 14]. The idea of a causal relation between severe heart failure leading to diabetes has, however, also been suggested by at least one interventional study showing that patients with very severe heart failure who were given a left ventricular assist device improved their diabetic control, and $6 / 15$ patients with diabetes were completely restored to a non-diabetic state [15].

Even though this study does not give any explanation of the mechanism underlying the development of diabetes in patients with heart failure, several potential mechanisms merit discussion. Patients with heart failure have decreased cardiac output and thereby diminished oxygen, glucose and insulin distribution to peripheral muscular tissue. Impaired blood flow further increases levels of adrenaline (epinephrine) and noradrenaline (norepinephrine). This neurohumoral compensating mechanism has been suggested to increase insulin resistance and hepatic gluconeogenesis as well as decrease the release of insulin from the pancreatic beta cells [16]. Sympathetic nervous system overactivity has also been shown to acutely reduce insulin sensitivity, but the role of development of overt diabetes remains to be established $[5,17,18]$. Patients with severe heart failure often lose muscle mass because of physical inactivity and/or cachexia/wasting, which increases insulin resistance $[19,20]$. Finally, although this is not a wellestablished side effect, loop diuretics may also contribute to impaired glucose tolerance per se, perhaps in a similar manner to that reported for thiazide diuretics [21].

Although the results of the present study should be regarded as hypothesis generating, it is interesting to note that RASis seemed to attenuate the risk of diabetes associated with severe heart failure. Large-scale clinical studies have found that treatment with RASis or angiotensin II receptor antagonists reduced the relative risk of the development of diabetes by $14-34 \%$ [22-27], although newer studies have found less or no risk reduction $[28,29]$. Other medications such as $\beta$ blockers have also been linked to increased risks of diabetes in some previous studies. We found the opposite in the present study, i.e. a lowered risk of diabetes associated with $\beta$ blockers, which, as for RASis, may have been due to blockage of a high sympathetic tone and/or improved cardiac function, although a selection bias of prescribing $\beta$ blockers to generally healthier patients cannot be excluded.

This study further emphasises the importance of identifying the diabetic subgroup of patients with heart failure, as they have worsened prognosis (demonstrated by this and other studies) [30]. Awareness of the increased risk of development of diabetes in patients with severe heart failure is therefore warranted. Initiatives to initiate early and more aggressive pharmacological interventions might be of value, but this needs further investigation.
Strengths and limitations This study was based on nationwide data. The Danish National Patients Registry includes discharge diagnoses of all Danish hospitals and therefore avoids selection bias. However, this was an observational study and The Danish National Patients Registry does not include clinical variables such as BMI, smoking status, blood samples including blood glucose values, NYHA class, hypertension and left ventricular ejection fraction. In particular, both heart failure severity and diabetes were defined from prescription claims, which are only rough phenotypic markers of true diabetes and heart failure severity. Finally, the study cohort only included patients who were hospitalised; thus patients with milder stages of heart failure may not have been included.

Conclusions This study, based on nationwide data, suggests an increased risk of development of diabetes in patients with heart failure, with increasing loop-diuretic dosage used as a proxy for heart failure severity. It emphasises the need to monitor and treat patients with heart failure to prevent diabetes development. Future strategies for heart failure management should include increased awareness of risk of diabetes in patients with severe heart failure.

Funding The study was funded by an internal research foundation grant from the Department of Cardiology, Gentofte Hospital. CA was funded by an independent research grant from the Danish Agency for Science, Technology and Innovation (grant number FSS - 11-120873). GHG was supported by an independent research scholarship from the Novo Nordisk Foundation.

Duality of interest AV has equity ownership in Novo Nordisk A/S and has received honoraria for lectures for Novo Nordisk A/S. GHG has stock ownership in Novo Nordisk A/S. All other authors declare that there is no duality of interest associated with their contribution to this manuscript.

Contribution statement MND wrote the initial draft of the manuscript. CA was responsible for data analyses and takes full responsibility for the accuracy of analyses and integrity of the data. All authors contributed to study design, interpretation of the data and critical revision of the manuscript. All authors approved the final manuscript.

\section{References}

1. Kannel WB, McGee DL (1979) Diabetes and cardiovascular disease. The Framingham study. J Am Med Assoc 241:2035-2038

2. From AM, Leibson CL, Bursi F et al (2006) Diabetes in heart failure: prevalence and impact on outcome in the population. Am J Med 119: 591-599

3. Cubbon RM, Adams B, Rajwani A et al (2013) Diabetes mellitus is associated with adverse prognosis in chronic heart failure of ischaemic and non-ischaemic aetiology. Diabetes Vasc Dis Res 10(4):330-336 
4. de Groote P, Lamblin N, Mouquet F et al (2004) Impact of diabetes mellitus on long-term survival in patients with congestive heart failure. Eur Heart J 25:656-662

5. Swan JW, Anker SD, Walton C et al (1997) Insulin resistance in chronic heart failure: relation to severity and etiology of heart failure. J Am Coll Cardiol 30:527-532

6. AlZadjali MA, Godfrey V, Khan F et al (2009) Insulin resistance is highly prevalent and is associated with reduced exercise tolerance in nondiabetic patients with heart failure. J Am Coll Cardiol 53:747-753

7. Nikolaidis LA, Sturzu A, Stolarski C et al (2004) The development of myocardial insulin resistance in conscious dogs with advanced dilated cardiomyopathy. Cardiovasc Res 61:297-306

8. Andersson C, Norgaard ML, Hansen PR et al (2010) Heart failure severity, as determined by loop diuretic dosages, predicts the risk of developing diabetes after myocardial infarction: a nationwide cohort study. Eur J Heart Fail 12:1333-1338

9. Kümler T, Gislason GH, Kirk V et al (2008) Accuracy of a heart failure diagnosis in administrative registers. Eur J Heart Fail 10:658-660

10. Gaist D, Sørensen HT, Hallas J (1997) The Danish prescription registries. Dan Med Bull 44:445-448

11. Schramm TK, Gislason GH, Køber L et al (2008) Diabetes patients requiring glucose-lowering therapy and nondiabetics with a prior myocardial infarction carry the same cardiovascular risk: a population study of 3.3 million people. Circulation 117:1945-1954

12. Fosbøl EL, Gislason GH, Jacobsen S et al (2009) Risk of myocardial infarction and death associated with the use of nonsteroidal antiinflammatory drugs (NSAIDs) among healthy individuals: a nationwide cohort study. Clin Pharmacol Ther 85:190-197

13. Gislason GH, Rasmussen JN, Abildstrøm SZ et al (2006) Long-term compliance with beta-blockers, angiotensin-converting enzyme inhibitors, and statins after acute myocardial infarction. Eur Heart J 27:1153-1158

14. Amato L, Paolisso G, Cacciatore F et al (1997) Congestive heart failure predicts the development of non-insulin-dependent diabetes mellitus in the elderly. The Osservatorio Geriatrico Regione Campania Group. Diabetes Metab 23:213-218

15. Uriel N, Naka Y, Colombo PC et al (2011) Improved diabetic control in advanced heart failure patients treated with left ventricular assist devices. Eur J Heart Fail 13:195-199

16. Kostis JB, Sanders M (2005) The association of heart failure with insulin resistance and the development of type 2 diabetes. Am J Hypertens 18:731-737

17. Reaven GM (1991) Insulin resistance, hyperinsulinemia, hypertriglyceridemia, and hypertension. Parallels between human disease and rodent models. Diabetes Care 14:195-202
18. Coats AJ, Anker SD, Anker S (2000) Insulin resistance in chronic heart failure. J Cardiovasc Pharmacol 35:S9-S14

19. Toth MJ, Gottlieb SS, Fisher ML, Poehlman ET (1997) Skeletal muscle atrophy and peak oxygen consumption in heart failure. Am J Cardiol 79:1267-1269

20. Tenenbaum A, Fisman EZ (2004) Impaired glucose metabolism in patients with heart failure: pathophysiology and possible treatment strategies. Am J Cardiovasc Drugs Drugs Devices Interv 4: 269-280

21. Zillich AJ, Garg J, Basu S et al (2006) Thiazide diuretics, potassium, and the development of diabetes: a quantitative review. Hypertension 48:219-224

22. Niklason A, Hedner T, Niskanen L, Lanke J (2004) Development of diabetes is retarded by ACE inhibition in hypertensive patients: a subanalysis of the Captopril Prevention Project (CAPPP). J Hypertens 22:645-652

23. Yusuf S, Sleight P, Pogue J et al (2000) Effects of an angiotensinconverting-enzyme inhibitor, ramipril, on cardiovascular events in high-risk patients. The Heart Outcomes Prevention Evaluation Study Investigators. N Engl J Med 342:145-153

24. The ALLHAT Officers and Coordinators for the ALLHAT Collaborative Research Group (2002) Major outcomes in high-risk hypertensive patients randomized to angiotensin-converting enzyme inhibitor or calcium channel blocker vs diuretic: the Antihypertensive and Lipid-Lowering Treatment to Prevent Heart Attack Trial (ALLHAT). J Am Med Assoc 288:2981-2997

25. Lindholm LH, Ibsen H, Borch-Johnsen K et al (2002) Risk of newonset diabetes in the Losartan Intervention For Endpoint reduction in hypertension study. J Hypertens 20:1879-1886

26. Lithell H, Hansson L, Skoog I et al (2003) The Study on Cognition and Prognosis in the Elderly (SCOPE): principal results of a randomized double-blind intervention trial. J Hypertens $21: 875-886$

27. Julius S, Kjeldsen SE, Weber M et al (2004) Outcomes in hypertensive patients at high cardiovascular risk treated with regimens based on valsartan or amlodipine: the VALUE randomised trial. Lancet 363:2022-2031

28. Bosch J, Yusuf S, Gerstein HC et al (2006) Effect of ramipril on the incidence of diabetes. N Engl J Med 355:1551-1562

29. NAVIGATOR Study Group, McMurray JJ, Holman RR, Haffner SM et al (2010) Effect of valsartan on the incidence of diabetes and cardiovascular events. N Engl J Med 362:1477-1490

30. Andersson C, Weeke P, Pecini R et al (2010) Long-term impact of diabetes in patients hospitalized with ischemic and non-ischemic heart failure. Scand Cardiovasc J 44:37-44 